POSTPRINT of the article published in 2019 in The Senses and Society, Vol. 14(1), 1-14.

\title{
Music, rowing, and the aesthetics of rhythm
}

\section{Jonna K. Vuoskoski}

RITMO Centre for Interdisciplinary Studies in Rhythm, Time and Movement, Department of Musicology \& Department of Psychology, University of Oslo, Norway

\begin{abstract}
Having 'good rhythm' is essential in both music and competitive rowing, but what exactly constitutes 'good rhythm', and how do we achieve it? Although rhythm is often discussed in purely auditory terms, I argue that rhythm is fundamentally a multisensory, kinaesthetic phenomenon. By drawing parallels between music and rowing, I illustrate how biological motion principles underlie the parameters of rhythm in both disciplines, and how the cognition and appreciation of rhythm is deeply embodied. I suggest that the two main ways in which rhythms generate pleasure in both music and rowing are by enabling behavioural synchrony between individuals, and by engaging the body in the cognitive process of rhythm perception and prediction. In essence, 'good rhythm' - a rhythm that is enjoyed and appreciated - is rhythm that moves.
\end{abstract}

Keywords: Rhythm; music; rowing; embodied cognition; synchronization; predictive coding

Rhythm is a fundamental feature of human actions such as locomotion, speech, and music. In this essay, I will consider the pleasure-evoking aspects of rhythm in two domains that are characterized by shared, recurring rhythmic patterns: music and competitive rowing. First, I will introduce the building blocks and main functions of rhythm in both disciplines, and describe some of the core processes involved in the coordination of joint action. Next, I will synthesize empirical findings linking synchronized joint actions with various positive outcomes, outlining the potential mechanisms underlying the effects of behavioural synchrony on pleasure. Finally, I will consider how rhythmic complexity may contribute to experiences of pleasure through the 
POSTPRINT of the article published in 2019 in The Senses and Society, Vol. 14(1), 1-14.

dynamics of anticipation and prediction error, and may even facilitate experiences of 'flow'.

\section{The building blocks of rhythm in music and rowing}

'Rhythm' can be roughly characterized as the temporal organisation of events, typically involving recurring patterns. In music and rowing, rhythm is a multi-layered phenomenon, where the timing patterns of events in the different layers vary in terms of timescale and complexity. In music, the most fundamental layer is an emergent property commonly called 'the beat', which is the regular, underlying pulse of music. Closely related to the beat is 'metre', which refers to the hierarchical organization of beats, in which some beats are perceived as stronger than others. These recurring hierarchical patterns are what generate the swinging feel of a waltz (strong-weak-weak) or the steady progression of a march (strong-weak-strong-weak). A metric cycle thus consist of multiple beats (commonly 2-4 in Western music) with varying strengths/emphases. Metrical systems are found in music cultures throughout the world, and range from simple duple and triple metres to more complex organisations (such as septuple metre, common in Balkan folk music). All rhythmic events - which can either emphasize or challenge the underlying metrical structure - are layered on top of this grid-like framework.

In rowing, the basic unit of action is the stroke cycle, which consists of the 'catch' (placement of the oar blade in the water), 'draw' (the propulsive phase), 'finish' (extraction of the oar blade from the water), and 'recovery', where the oar is moved back to catch position above the surface of the water. In modern racing boats the rower's seat is set on rails, enabling the rower to move backwards and forwards during the draw and recovery phases (respectively) to maximise the length of the stroke. Just as musical tempo is typically described in the form of beats per minute (bpm), rowing tempo is expressed in terms of strokes per minute (spm). However, in terms of timescale and conceptualisation, the stroke cycle is perhaps more analogous to a metric cycle (discussed in the previous paragraph) than a beat. In rowing, typical stroke rates vary between 18 and $40 \mathrm{spm}$, while in Western popular music the most common tempo is in the range of 
POSTPRINT of the article published in 2019 in The Senses and Society, Vol. 14(1), 1-14.

100-120 bmp (Moelants et al., 2002; van Buskirk, 2013). In crew boats, the stroke rate is determined by the rower sitting closest to the stern of the boat, the 'stroke', who sets the rhythm followed by the rest of the crew. Although rhythm (in terms of the stroke rate and the timing ratio of the draw and recovery phases) is crucial in single sculling as well, being in synchrony and moving together with others is of paramount importance for efficient boat propulsion in crew boats.

The stroke rower also determines the relative durations of the draw and recovery phases and the specific timing of the catch and finish points, which have to remain consistent in order to provide a predictable pattern for the other rowers to follow. In contrast to most musical metres, which tend to have isochronous beats (i.e., beats of equal duration), the different parts of the rowing stroke cycle have asymmetrical - yet consistent - durations. However, non-isochronous musical metres exist as well, such as the traditional Norwegian Telespringar style, which has a three-beat metric cycle with a long-mediumshort pattern (Haugen, 2016). Thus, a useful way of conceptualising the rhythm of rowing could be to characterize it in terms of non-isochronous metre, with the stroke cycle corresponding to a metric cycle. The rhythm of the stroke cycle is further constrained by biological motion principles and boat speed, which affect the speed of movement during both the draw and recovery phases. Similarly to rowing, the tempo and rhythm in music are also constrained by biological motion principles specific to the human body, with the average preferred tempo of music (around 100-120 bpm; Moelants, 2002; van Buskirk, 2013) - corresponding to the central "resonant frequency" of human movement observed in spontaneous human locomotion (MacDougall \& Moore, 2005). Moreover, timing features such as ritardandos (i.e., the gradual deceleration of the tempo) in the end of musical pieces have been found to follow a mathematical function similar to that of runners' decelerations (Friberg \& Sundberg, 1999), for example. Thus, the rhythm and tempo in both music and rowing are underpinned and shaped by biological motion.

An important function of rhythm in both music and rowing is to enable prediction and thus synchronization of joint action. In both music-making and rowing, interpersonal synchronisation is enabled by shared frames and knowledge structures, yet still involves 
POSTPRINT of the article published in 2019 in The Senses and Society, Vol. 14(1), 1-14.

constant adaptation to others' timing. Indeed, synchronisation in the absence of accurate prediction of future events would be practically impossible. In music, a fundamental predictive structure is provided by the meter, in which each musical time-point encompasses a conjoint prediction of timing and strength (Vuust \& Kringelbach, 2010). Although the perception of beat and metre in music are largely influenced by objective features such as loudness and salience, style-specific knowledge, acquired through cultural exposure and statistical learning, also plays a role: For example, when asked to tap to the beat of South African and European folk songs, European and South African participants tend to differ in terms of their consensus regarding the location of the beat (Toiviainen \& Eerola, 2003). This example illustrates that - even though the neural mechanisms of beat perception are shared by all humans - the emergence of shared perceptions and predictions requires shared knowledge structures. The same is true for rowing, where millisecond-accurate synchronisation is achieved through shared mental models of the ideal stroke cycle, acquired through extended periods of joint practice (cf. King \& de Rond, 2011).

While having shared predictive models could be seen as a prerequisite for successful synchronisation of joint action, it is not sufficient by itself: Accurate synchronisation is achieved and fine-tuned through multisensory cues of others' actions, and by adjusting one's own motor actions accordingly (i.e., sensorimotor synchronisation). In the context of music-making and rowing, such cues can be auditory (e.g., sounds produced by playing musical instruments, the sound of oars hitting the water), visual (gestures, body motion), and vestibular/somatosensory (motion felt through the hull of the boat, vibrations of sound waves felt in the body), for example. Recent findings from research using transcranial magnetic stimulation (TMS) have revealed that motor simulation seems to play an important role in the temporal coordination of (inter)actions (Hadley et al., 2015). TMS is a non-invasive method of altering brain activity, which can either activate or inhibit neurons in a specific target region. Hadley and colleagues (2015) found that pianists used internal motor simulation of their partner's part to predict their timing patterns in a musical turn-taking task: When the brain regions associated with motor resonance and simulation were inhibited, the pianists' timing coordination became less 
POSTPRINT of the article published in 2019 in The Senses and Society, Vol. 14(1), 1-14.

accurate. Similar motor resonance - where the coordination of movements is enabled by internal simulation - is thought to take place during synchronized actions, with the added element of the same neural pathways being utilized by both observed and performed actions, resulting in partial overlap of the neural representations of the self and the other (e.g., Hove \& Risen, 2009). Furthermore, since all music has traditionally been produced by (human) bodily action, it is thought that one of the ways we make sense of musical expression - within and across cultures - is through internal simulation: Perceived physical energy in sound is converted into 'action-oriented' meanings through correspondence with bodily movement and gesture (Leman, 2007; Molnar-Szakacs \& Overy, 2006). Moreover, it has been argued that we also perceive gestural properties in sounds not produced by human bodily action, and use motor cognition to make sense of environmental and synthesized sounds as well (e.g., Godøy et al., 2016).

\section{Benefits of synchronized behaviour}

Synchronous actions have been associated with subsequent increases in positive affect (e.g., Tschacher, Rees, \& Ramseyer, 2014). Interestingly, moving in synchrony with others has also been shown to have a multitude of pro-social effects, facilitating cooperation, social bonding, empathy, and helping behaviour (e.g., Hove \& Risen, 2009; Kirschner \& Tomasello, 2010; Rabinowitch et al., 2013; Tarr et al., 2015; Wiltermuth \& Heath, 2009). Communal music-making seems to be a particularly effective for forming social bonds quickly: A study comparing choir singers and attendants of non-musical group activities (creative writing and crafts) demonstrated that the choir singers started to feel closer to their newly formed group significantly more quickly than the attendants of the other two activities (Pearce et al., 2015). Even very simple manipulations of synchronicity in tasks such as tapping and walking with others have been shown to affect

levels of cooperation and feelings of affiliation, with synchronous actions leading to more pro-social behaviour (e.g., Hove \& Risen, 2009; Wiltermuth \& Heath, 2009). This positive association between synchronous actions and prosocial behaviour seems to emerge very early in development, and has been documented in infants as young as 14 months old (Cirelli et al., 2014). Consequently, some have proposed that music-making may have offered an evolutionary advantage by promoting social bonding and group 
POSTPRINT of the article published in 2019 in The Senses and Society, Vol. 14(1), 1-14.

cohesion through behavioural synchrony (e.g., Brown, 2000; Cross \& Morley, 2008; Freeman, 2000; Huron, 2001; Tarr, Launay, \& Dunbar, 2014). The hypothesized mechanisms underlying these effects include self-other merging (where the neural representations of self- and other-produced actions closely overlap; cf. Hove \& Risen, 2009), shared intentionality (e.g., Reddish et al., 2013), and endorphin release (e.g., Tarr et al., 2014, 2015).

Empirical evidence for endorphin release in the context of behavioural synchrony comes from studies demonstrating increased pain thresholds after synchronous vs. asynchronous (Tarr et al., 2015) and in-phase vs. antiphase actions (Sullivan et al., 2014). Increased pain tolerance is of significant importance for a competitive sport like rowing, as this might enable sustaining higher levels of effort for longer periods of time. Indeed, Cohen and colleagues (2009) demonstrated that rowers had higher pain thresholds after rowing in synchrony with others (on rowing machines) compared to rowing alone. Sullivan and colleagues (2014) further extended these findings by showing that increased pain thresholds are specific to in-phase synchronous rowing. In addition to potentially boosting performance through increased pain tolerance, behavioural synchrony seems to reduce the actual effort required to carry out movements. In a study involving cycling either synchronously or asynchronously to the beat of accompanying music, Bacon and colleagues (2012) demonstrated that synchronous cycling was associated with lower oxygen consumption. Thus, in addition to making the boat move more effectively, behavioural synchrony actually makes rowers row more efficiently, and enables them to sustain higher levels of effort for longer.

\section{Rhythmic complexity, and the experiences of 'groove' and 'flow'}

Crucially, the aesthetics of 'good rhythm' go beyond mere synchronization. In rowing, rhythmic complexity emerges from the time ratio between the drive and recovery phases of the rowing stroke, which is key to optimal boat propulsion. More specifically, the speed of body movement during the recovery phase - where the centre of mass of the rowers moves in the opposite direction in relation to the motion of the boat - has to be proportional to the speed of the boat in order to minimise boat deceleration. This optimal 
POSTPRINT of the article published in 2019 in The Senses and Society, Vol. 14(1), 1-14.

draw/recovery ratio, combined with precise inter-rower synchronization, constitute the elusive 'good rhythm' or 'swing' that rowing crews strive for. Here, the speed, acceleration and deceleration of body movements are as integral to the notion of rhythm as the timing of those movements. Succeeding or failing to find 'the rhythm' can either make or break a race, as illustrated by a case study of the 2007 Oxford and Cambridge Boat Race by King and de Rond (2011). They identified a specific moment after the first quarter of the race, where the Cambridge crew, after struggling to find their rhythm for the first part of the race, managed to reset their rhythm into a 'good' one, resulting in their eventual victory over Oxford. In essence, good rhythm in rowing is constituted by both temporal and kinaesthetic properties, and has a propulsive yet 'effortless' and flowing quality.

In music, the emergent, propulsive qualities often called 'groove' and 'swing' are generated by optimally alternating patterns of 'off-beat' and 'on-beat' rhythms, and by micro-deviations from the metrical grid. The feeling of 'groove' is characterized by a feeling of pleasure and a desire to move to the beat of the music. There is mounting evidence suggesting that - even in the absence of actual bodily movement - the cognition of rhythm and metre is deeply embodied: Studies investigating the neural processes involved in music listening have demonstrated that the brain regions involved in motor planning and execution play an important role in rhythm and beat processing (e.g., Grahn \& Brett, 2007; Grahn, 2012). More recent evidence for the embodied cognition of rhythm and metre comes from a study by Stupacher and colleagues (2013) that investigated the activity within the motor system (motor-evoked potentials, measured using TMS and electrodes placed on muscles in the forearm) in response to low-groove and high-groove musical examples (categorized on the basis of previously collected ratings of 'grooviness'). They found that musically trained participants exhibited higher MEPs in response to high-groove (vs. low-groove) music, especially when the TMS pulse was administered on the beat (rather than off the beat), suggesting that high-groove music engages the motor system to a more significant degree than low-groove music. These findings provide empirical support for the notion that we understand groove through our bodies (cf. Roholt, 2014). 
POSTPRINT of the article published in 2019 in The Senses and Society, Vol. 14(1), 1-14.

One promising approach to understanding the pleasure associated with the feeling of 'groove' is through the predictive coding theory of cognition (e.g., Vuust \& Witek, 2014), which emphasizes the central role of constant predictions generated and updated by the brain in informing perception and cognition (Clark, 2013). Witek and colleagues (2014) investigated the role of rhythmic complexity and syncopation (i.e., rhythmic patterns that fall 'off' the beat) in eliciting pleasure and the desire to move to music, and found an inverted U-relationship between the amount of syncopation and experienced groove. Witek and colleagues (2014) proposed that moderate amounts of syncopation increase rhythmic ambiguity without breaking down the underlying, predicted metric structure, and that bodily movement (overt or simulated) is used to enact the beat in order to increase perceptual fluency. The cognitive process of beat perception and prediction is, in a sense, extended to the body, while the 'dissonance' or 'gap' between the predicted metric hierarchy and the perceived rhythmic syncopation is what leads to greater arousal and greater pleasure. In other words, rhythms with low degrees of syncopation are not experienced as arousing due to their high predictability, while rhythms with too high degrees of syncopation actually prevent the generation of metric predictions: When too many rhythmic events occur off-beat rather than on-beat, beat perception (and prediction) starts to shift and becomes unstable, eventually breaking down. However, when the predicted metric model and accurate beat prediction are sustained in the face of increased uncertainty (generated by syncopation), the positive prediction error (i.e., correctly predicting something on the basis of uncertain odds; cf. Schultz, 2016) leads to increased dopamine release in the brain, and increased feelings of pleasure.

Similar accounts have been offered for the pleasure generated by musical expectations in general (e.g., Huron, 2006; Meyer 1956), and PET-imaging studies have found evidence of dopamine release associated with musical anticipation (Salimpoor et al., 2011). It may be that our brains actually reward the generation of predictions, as this is what leads to learning and the generation of more accurate predictions in the future (Clark, 2013; Vuust \& Witek, 2014). In any case, there is mounting evidence showing that in the case of groove, the body plays an important role in the generation of metric predictions and the 
POSTPRINT of the article published in 2019 in The Senses and Society, Vol. 14(1), 1-14.

ensuing feelings of pleasure. An illustrative example is offered by the genre of electronic dance music (EDM), where pieces typically follow the same formula consisting of a 'breakdown', a 'build-up' and a 'drop' of the beat, playing with - and capitalizing on the listeners'/dancers' predictions and anticipation. The 'drop' of the beat (i.e., when the bass and beat return in their full intensity) is characterized by increased synchronous bodily movement and feelings of pleasure (e.g., Solberg \& Jensenius, 2017); in other words, an experience of 'groove', made all the stronger by the anticipation and fulfilment of predictions.

In both instances - music and rowing - the experiences of groove and propulsion can be highly pleasurable, but there is yet another way in which rhythmic complexity and sensorimotor synchronisation can contribute to feelings of pleasure: By facilitating a state of 'flow'. Flow states typically occur in situations where challenging tasks are met with high levels of skill, and are characterized by positive affect, concentration, creativity, motivation, and satisfaction (Csikszentmihalyi \& LeFevre, 1989). Indeed, musical and athletic performance are some of the most common scenarios for experiences of flow to occur in (for reviews, see Chirico et al., 2015; Swann et al., 2012). Intriguingly, recent research has associated the experience of flow states with the accuracy of sensorimotor synchronisation (i.e., tapping to the beat of music) - especially in the case of more complex rhythms (Stupacher et al., 2017). Nevertheless, it is not yet well understood which rhythmic properties and processes might facilitate flow states (or how). It could be postulated, however, that rhythmic complexity and synchronisation might be some of the key elements that contribute to experiences of flow in the context of both music and rowing: Finding 'good rhythm' in rowing, or being 'in the groove' when playing (or dancing to) music certainly requires accurate prediction and sensorimotor integration, and often involves complex rhythmic phenomena. Moreover, finding 'the rhythm' or 'swing' in rowing, or being 'in the groove' in music are also associated with increased fluency one of the defining characteristics of a flow state.

\section{Conclusion}


POSTPRINT of the article published in 2019 in The Senses and Society, Vol. 14(1), 1-14.

Considering the cognition and appreciation of rhythm in two different disciplines - music and rowing - contributes to the understanding and conceptualisation of rhythm as a domain-general phenomenon. Although musical rhythm has traditionally been theorized in purely auditory terms - and rowing rhythm in terms of movement - more careful consideration reveals that both phenomena are fundamentally multisensory and grounded in bodily motion. Indeed, the process of rhythm cognition has been shown to be deeply embodied, involving motor simulation and resonance. Furthermore, both music and rowing are characterized by shared rhythms that underpin accurate prediction and synchronization of joint action. These shared rhythms have their bases in shared knowledge structures that are acquired through cultural exposure and joint practice.

The two main ways in which rhythm contributes to feelings of pleasure - by facilitating behavioural synchrony, and by affording arousing experiences of 'prediction error' - are characterised by conformity and disruption, respectively. This apparent contradiction can be reconciled by considering the underlying mechanisms that can function in an independent yet complementary manner. Shared rhythms and frameworks enable coordinated joint action and also facilitate cooperation, and thus it is plausible that the capacity for behavioural synchrony may have offered an evolutionary advantage for early humans. If specific reward mechanisms (e.g., endorphin release) are indeed triggered by behavioural synchrony (as suggested by preliminary empirical findings; e.g., Sullivan et al., 2014; Tarr et al., 2015), this would explain why synchronized joint actions such as rowing or music-making are experienced as pleasurable.

The pleasure of 'disruption' emerges from rhythmic complexity and the dynamics of anticipation and prediction error. Furthermore, the involvement of the motor system in beat processing highlights the action-based cognition of rhythm. Especially when rhythmic complexity increases and beat clarity decreases, bodily action (or the simulation of bodily action) functions as an 'enactment' of the predicted metric structure, aiding and informing perception. Building on recent developments in the predictive coding theory of cognition, the pleasurable experiences 'groove' - arising from optimal rhythmic complexity and unpredictability - could be understood as a case of positive prediction 
POSTPRINT of the article published in 2019 in The Senses and Society, Vol. 14(1), 1-14.

error. In positive prediction error, the brain rewards correct predictions more (i.e. more dopamine is released) when those predictions are made on the basis of uncertain rather than certain odds (e.g., Schultz, 2016). Thus, in the case of groove, pleasure is experienced when the incoming sensory information about rhythmic events is relatively uncertain, but the underlying metric structure is nevertheless correctly predicted - with the help of motor action and/or simulation.

Finally, accurate sensorimotor integration and synchronisation can contribute to the experience of 'flow'; a state of concentrated engagement associated with feelings of effortlessness and pleasure. The occurrence of flow typically increases with task difficulty coupled with expertise, and thus complex actions requiring training and sensorimotor accuracy - like rowing or music-making - are optimal for fostering such experiences. Even music listening - particularly in the case of optimally complex, 'groovy' music that invites sensorimotor synchronisation (i.e., dancing) - can bring about such experiences through increased perceptual fluency in the face of rhythmic uncertainty.

\section{Acknowledgments}

I would like to thank the members of the Leverhulme International Network 'Evaluating Methods of Aesthetic Enquiry across Disciplines' for their valuable comments on an earlier version of this paper. I would also like to thank the two anonymous reviewers whose insightful and helpful suggestions helped to improve this manuscript. 
POSTPRINT of the article published in 2019 in The Senses and Society, Vol. 14(1), 1-14.

\section{Response: Sensations of Movement and Embodied Cognition}

\section{Dee Reynolds}

French Studies, University of Manchester

Jonna Vuoskoski's discussion of rhythm is enlightening and is especially intriguing as it shows how the aesthetic principles of rhythm operate in rowing, which is defined by the OED not as an art but a 'sport' or 'pastime'. Indeed, rhythm may be regarded as linking the formal principles that characterise established art forms with activities and artefacts that are not culturally designated as 'art' but can nonetheless be considered 'aesthetic'. Moreover, by showing how complex rhythms can engage the motor system, thereby involving bodily experience, Vuoskoski's argument points to how embodied cognition as well as pleasure is key to the aesthetics of rhythm. Although the term 'aesthetic' is frequently used almost interchangeably with 'pleasurable', a key element of aesthetic

pleasure is that 'bodily experience informs cognition and is even an inseparable part of cognition' (Vuoskoski, p.1). Rhythm is perhaps the best example of how knowledgebased aesthetic pleasure is generated and experienced, and this is reflected in the fact that different art forms, in diverse times and places, have devised rhythmic 'rules' that are specific to their own medium and practices.

I would like to develop this discussion to explore further how the aesthetics of rhythm involves knowledge that is grounded in the senses and how this is linked with complexity. Sensible (sensory) cognition was considered fundamental to aesthetics by Alexander Gottlieb Baumgarten (1714-1762). Baumgarten was the author of Aesthetica, the first volume of which appeared in 1750, but he had already discussed key themes in his reflections on poetry, Meditationes Philosophicae de Nonnullis ad Poema Pertinentibus ["Philosophical meditations pertaining to some matters concerning poetry"] (1735). Here he introduced the term 'aesthetics' as the treatment of sensate subject matter, or the 'science of perception' (Baumgarten, Meditationes, par. 116), which he later described in Aesthetica as 'the science of sensible cognition' (par. 1; cited Guyer 2016). Unlike philosophical knowledge that was considered to be based on 'distinct' 
POSTPRINT of the article published in 2019 in The Senses and Society, Vol. 14(1), 1-14.

notions or concepts, sensible cognition was described as 'confused', meaning that it did not have precise boundaries that demarcated separate concepts (Meditationes, par. 14). Sensory cognition could be extended and made more complex when different concepts were linked together (par. 23), and Baumgarten argued that 'the more extensive clarity the representation has, the more poetic it is' (par. 18). Complexity, then, was a key element of the sensory cognition that was particular to poetics and aesthetics.

Vuoskoski's discussion of the aesthetics of rhythm shows how its pleasures are linked with learning and sensory cognition that is enacted in the body. She argues that experiencing rhythm is a source of pleasure, in two potential ways. The first is through shared rhythm, or synchronisation of joint action, and the second is through prediction, which becomes pleasurable where an optimal degree of complexity brings bodily action (movement) into play. The first kind of pleasure can be seen as a form of social reward, which may have evolved because behavioural synchrony is advantageous to the success of the human species. It has been shown that moving together reduces effort and energy expenditure. The second kind of pleasure is linked with what Vuoskoski calls, in her closing words, 'increased perceptual fluency in the face of rhythmic uncertainty'. Heightened levels of skill and performance give rise to a feeling of effortlessness, which is experienced as pleasurable. Rhythm, then, can lead both to feelings of social cohesion and of increased physical power, where the same movement can be accomplished with less effort.

The rhythmic functions that Vuoskoski highlights - synchronization and prediction involve learning and forms of knowledge that are enacted in movement, whether actual or simulated. To achieve movement that is co-ordinated with others requires a certain adaptation or attunement. Through attention to multisensory cues (visual, auditory and proprioceptive), participants in rhythmic actions adapt their behavior to each other. This kinesthetic sensing leads to changes in behavior where self-other boundaries can be blurred and new ways of interacting with the others and the world can be discovered. Often, co-ordinating actions with others is multi-layered and complex, as different organizational principles are combined, such as sets of rules pertaining to the activity in 
POSTPRINT of the article published in 2019 in The Senses and Society, Vol. 14(1), 1-14.

question, the group of participants and in some instances a leader (e.g. the stroke cycle in rowing, the rowers, and the 'stroke' sitting closest to the stern).

It is not only active participants who can experience kinesthetic engagement. Vuoskoski discusses how listening to music can also engage the motor system, and much research has been carried out on dance spectators, showing how looking at others dance can 'move' those who watch, physiologically as well as emotionally, through kinesthetic empathy (e.g. Jola 2012; Reason 2010). Whether or not they lead to actual movement, rhythmic patterns of repetition and variation can activate motor planning by creating anticipation, putting our bodies in gear to move, or preparing us for the next move. When a group of different individuals are listening to music or watching dance, they are responding to the same rhythmic stimulus and their motor systems may be activated in similar ways, even if they are not aware of the others, though their responses are also influenced by culturally shared knowledge.

Vuoskoski argues that in listening to music, it is particularly when rhythms are complex that they activate the movement sense - 'high-groove music engages the motor system to a more significant degree than low-groove music' (p.6). Engaging the body in movement or in preparing to move enables us to make sense of what is happening, reduces prediction error and leads to a state of 'flow'. This rhythmic response brings new levels of understanding that emerge in the body. The pleasure afforded by such experiences can be described as 'aesthetic' in that it involves not only sensation, but also increased knowledge.

Bodily action - which I shall simply call movement - plays a crucial role in the aesthetics of rhythm across art forms. Different art forms have developed specific ways of organising the material of their medium to produce rhythmic effects and elicit motor responses that frequently carry semantic content. This means that without actually moving, people engaging with these rhythmically ordered works can experience embodied senseations of movement. In poetry, for instance, metre and rhythm can 
POSTPRINT of the article published in 2019 in The Senses and Society, Vol. 14(1), 1-14.

produce sensations of speeding up and slowing down. In French poetry, the metrical tradition is for lines to contain the same number of syllables. The classical line, the alexandrine, contains twelve syllables with a conventional break at the sixth syllable (caesura). Within this regular, symmetrical structure, the arrangement of words within and across lines can lead to variations that are embodied in the breath and voice if spoken aloud. For instance, the line from the poem by Victor Hugo, 'Un jour je vis' ['One day I saw'] (Les Contemplations, 1856), 'Un rapi/de navir//e envelo/ppé de vents' ['a fast ship enveloped in winds']. This line describes a boat being blown along at speed. We do not pause at the central point of the line, the caesura, as it occurs within the word 'navire', but rush forward. The following two 'e' sounds blend into one, again increasing speed, and the other rhythmic divisions in the line also occur inside words, 'rapi/de' and 'envelo/ppé' (this is known as 'coupe enjambante'), accelerating the pace. This contrasts with the slower progression of the next line: 'Et j'entendis, penché //sur l'abîme des cieux' ['And I heard, leaning over the abyss of the heavens']. Here, the use of the comma and the fully pronounced 'e' at the caesura ('penché') and also in 'abîme' mean that individual sounds are given maximum value and the reading time is prolonged, heightening the pregnancy of the moment where the poet looks down upon the immensity of the abyss. Sometimes choices about where rhythmic divides are placed can shift both rhythm and meaning. For instance, if 'sur l'abîme des cieux' is read as 'sur l'abî/me des cieux' $(3+3)$, the mute ' $e$ ' at the end of 'abîme' is carried forward into the image of the heavens, creating a sense of sound travelling into infinite space. In the reading 'sur l'abîme/ des cieux' (4+2), on the other hand, the break comes after the mute 'e' (a 'coupe lyrique'), meaning that the sound is contained and held back. The former reading may be described as more kinaesthetic and the reader may empathize with the travelling motion of the sound into space, but the latter is also experienced proprioceptively, as a sense of containment. We can in fact feel both contrasting possibilities together, which defies logic but can be experienced kinesthetically, as sensory cognition. As in rowing and listening to music, poetic rhythm brings into play levels of understanding that are kinesthetic rather than purely cognitive. 
POSTPRINT of the article published in 2019 in The Senses and Society, Vol. 14(1), 1-14.

Also like rowing, and music-making/listening, poetry is a temporal art, since speaking, listening, writing and reading take place in time. However, it can also be experienced as a spatial art, whose rhythms unfold in space-time, allowing for multi-sensory correspondences between the textures of its language and those of its fictive spaces. In visual art, conventional techniques of inducing in the viewer feelings of movement in time include the use of converging lines and angles, or gradual gradations of colour, which invite the viewer to anticipate progression. Among the best-known attempts to create kinaesthetic sensations in painting took place in Futurism, notably Giacomo Balla's 'Dynamism of a Dog on a Leash' (1912). Here, the sensation of movement is dependent on our recognition of how dogs and people look while walking. However, movement sensations can be induced more directly, through perceiving rhythmic properties of the medium. As in music, complex pictorial rhythms that pose a challenge to the spectator can recruit kinesthetic sensation as a mode of embodied understanding and anticipation. Extreme examples of how complex visual rhythms in the pictorial medium engage our movement sense are found in the late work of Piet Mondrian (18721944). Mondrian's Boogie-Woogie pictures have been described in musical terms (as indeed the titles invite), and also in terms of the layout of New York streets, intersections, traffic lights, and flashing neon lights. Here, the rhythmic structures of the medium itself set patterns of expectation and interweave rhythmic events whose complexity engages our kinesthetic sense in navigating uncertainty.

Mondrian's last picture, 'Victory Boogie-Woogie' (1943-44: https://en.wikipedia.org/wiki/Victory Boogie Woogie), follows directly on his 'Broadway Boogie-Woogie' (1942-3). It preserves his classic building blocks of rectangles and primary colours, but its diamond format and asymmetrical composition create instability, and rectangles fragment into tiny cubes and cubes form rectangular and linear configurations that are in turn broken up. The balance between predictability and ambiguity is highly complex and engages our motor system in attempting to find a 'path' through the picture, which is constantly defeated: our gaze is drawn across the surface at different speeds and in continually changing directions, thereby enabling the picture's pulsating rhythms to be felt kinesthetically (Reynolds 1995). 
POSTPRINT of the article published in 2019 in The Senses and Society, Vol. 14(1), 1-14.

In conclusion, by bringing together discussion of rowing with music making and listening, Vuoskoski demonstrates the involvement of human bodily movement in rhythmic activity and response, and its role in embodied cognition that is itself pleasureinducing. This discussion goes to the heart of aesthetics, and relates to the history of the discipline. It also resonates across different art forms where complex rhythms invoke kinesthetic responses in order to 'make sense' of multi-layered meanings. Whether it is physically enacted, as in rowing, or virtually, as in listening, reading, or looking, rhythmic movement is a core element of kin/aesthetic experience.

\section{Author Biographies:}

Jonna Vuoskoski is Associate Professor in Music Cognition at the University of Oslo, Norway, where she is part of the RITMO Centre for Interdisciplinary Studies in Rhythm, Time, and Motion. Her main areas of interest are music-induced emotion, empathy, and the social and embodied cognition of music. She also rows competitively. Her recent work has been published in the journals Psychology of Music, Music Perception, Physics of Life Reviews, Emotion, and Frontiers in Psychology.

Dee Reynolds (French Studies, University of Manchester)

Dee Reynolds's research background is in French poetry, abstract painting and comparative aesthetics. Recent research has focussed on modern and contemporary dance, especially dance audiences and kinaesthetic empathy. 
POSTPRINT of the article published in 2019 in The Senses and Society, Vol. 14(1), 1-14.

\section{References}

Bacon, C. J., T. R. Myers, and C. I. Karageorghis. 2012. "Effect of music-movement synchrony on exercise oxygen consumption." Journal of Sports Medicine and Physical Fitness 52(4): 359-365.

Baumgarten, Alexander Gottlieb. 1954. Meditationes Philosophicae de Nonnullis ad Poema Pertinentibus. Transl. K. Aschenbrenner and W.B. Holther, Reflections on Poetry: Alexander Gottlieb Baumgarten's Meditationes philosophicae de nonnullis ad poema pertinentibus. Berkeley: University of California Press.

Brown, Steven. 2000. "Evolutionary models of music: From sexual selection to group selection." In Perspectives in ethology, edited by F. Tonneau \& N. S. Thompson, 231-281. Boston, MA: Springer.

Chirico, Alice, Silvia Serino, Pietro Cipresso, Andrea Gaggioli, and Giuseppe Riva. 2015. "When music "flows". State and trait in musical performance, composition and listening: a systematic review." Frontiers in Psychology 6: 906.

Cirelli, Laura K., Kathleen M. Einarson, and Laurel J. Trainor. 2014. "Interpersonal synchrony increases prosocial behavior in infants." Developmental Science 17 (6): 1003-1011.

Clark, Andy. 2013. "Whatever next? Predictive brains, situated agents, and the future of cognitive science." Behavioral and Brain Sciences 36 (3): 181-204.

Cohen, Emma E.A., Robin Ejsmond-Frey, Nicola Knight, and Robin I.M. Dunbar. 2009. "Rowers' high: behavioural synchrony is correlated with elevated pain thresholds." Biology Letters 6: 106-108.

Cross, Ian, and Iain Morley. 2009. "The evolution of music: Theories, definitions and the nature of the evidence." In Communicative Musicality: Exploring the Basis of Human Companionship, edited by S. Malloch \& C. Trevarthen, 61-81. Oxford, UK: Oxford University Press.

Csikszentmihalyi, Mihaly, and Judith LeFevre. 1989. "Optimal experience in work and leisure." Journal of Personality and Social Psychology 56 (5): 815-822.

Freeman, W. J., III. 2000.” A neurobiological role of music in social bonding”. In The Origins of Music, edited by N. Wallin, B. Merker \& S. Brown, 411-424. Cambridge, MA: MIT Press. 
POSTPRINT of the article published in 2019 in The Senses and Society, Vol. 14(1), 1-14.

Friberg, Anders, and Johan Sundberg. 1999. "Does music performance allude to locomotion? A model of final ritardandi derived from measurements of stopping runners". Journal of the Acoustical Society of America 105 (3): 1469-1484.

Godøy, Rolf Inge, Minho Song, Kristian Nymoen, Mari Romarheim Haugen, and Alexander Refsum Jensenius. 2016. "Exploring sound-motion similarity in musical experience." Journal of New Music Research 45 (3): 210-222.

Grahn, Jessica A. 2012. "Neural mechanisms of rhythm perception: current findings and future perspectives." Topics in Cognitive Science 4 (4): 585-606.

Grahn, Jessica A., and Matthew Brett. 2007. "Rhythm and beat perception in motor areas of the brain." Journal of Cognitive Neuroscience 19 (5): 893-906.

Guyer, Paul. 2016. "18th Century German Aesthetics". In The Stanford Encyclopedia of Philosophy (Winter 2016 Edition), edited by Edward N. Zalta. Retrieved from: https://plato.stanford.edu/archives/win2016/entries/aesthetics-18th-german/

Hadley, Lauren V., Giacomo Novembre, Peter E. Keller, and Martin J. Pickering. 2015.

"Causal role of motor simulation in turn-taking behavior." Journal of Neuroscience 35 (50): 16516-16520.

Haugen, Mari Romarheim. 2016. "Investigating periodic body motions as a tacit reference structure in Norwegian telespringar performance." Empirical Musicology Review 11 (2-3): 272-294.

Hove, Michael J., and Jane L. Risen. 2009. "It's all in the timing: Interpersonal synchrony increases affiliation." Social Cognition 27 (6): 949-960.

Huron, David. 2001. "Is music an evolutionary adaptation?" Annals of the New York Academy of Sciences 930 (1): 43-61.

Huron, David. 2006. Sweet Anticipation: Music and the Psychology of Expectation. Cambridge, MA: MIT press.

Jola, Corinne, Ali Abedian-Amiri, Annapoorna Kuppuswamy, Frank E. Pollick, and Marie-Hélène Grosbras. 2012. "Motor simulation without motor expertise: enhanced corticospinal excitability in visually experienced dance spectators." PloS One 7 (3): e33343.

King, Anthony, and Mark de Rond. 2011. "Boat race: rhythm and the possibility of collective performance." The British Journal of Sociology 62 (4): 565-585. 
POSTPRINT of the article published in 2019 in The Senses and Society, Vol. 14(1), 1-14.

Kirschner, Sebastian, and Michael Tomasello. 2010. "Joint music making promotes prosocial behavior in 4-year-old children." Evolution and Human Behavior 31 (5): 354-364.

Leman, Marc. 2007. Embodied Music Cognition and Mediation Technology. Cambridge, MA: MIT Press.

MacDougall, Hamish G., and Steven T. Moore. 2005. "Marching to the beat of the same drummer: the spontaneous tempo of human locomotion." Journal of Applied Physiology 99 (3): 1164-1173.

Meyer, Leonard. B. (1956). Emotion and Meaning in Music. Chicago, IL: University of Chicago Press.

Moelants, Dirk. 2002. "Preferred tempo reconsidered." In Proceedings of the 7th International Conference on Music Perception and Cognition, edited by C. Stevens, D. Burnham, G. McPherson, E. Schubert, and J. Renwick, 580-583. Sydney, Australia. Retrieved from: http://www.ipem.ugent.be/dirkmoelants/pub/ Moelants_PreferredTempo Reconsidered.pdf

Molnar-Szakacs, Istvan, and Katie Overy. 2006. "Music and mirror neurons: from motion to'e'motion." Social Cognitive and Affective Neuroscience 1 (3): 235241.

Pearce, Eiluned, Jacques Launay, and Robin I.M. Dunbar. 2015. "The ice-breaker effect: singing mediates fast social bonding." Royal Society Open Science 2 (10): 150221 .

Rabinowitch, Tal-Chen, Ian Cross, and Pamela Burnard. 2013. "Long-term musical group interaction has a positive influence on empathy in children." Psychology of Music 41 (4): 484-498.

Reason, Matthew, and Dee Reynolds. 2010. "Kinesthesia, empathy, and related pleasures: An inquiry into audience experiences of watching dance." Dance Research Journal 42 (2): 49-75.

Reddish, Paul, Ronald Fischer, and Joseph Bulbulia. 2013. "Let's dance together: synchrony, shared intentionality and cooperation." PloS One 8 (8): e71182.

Reynolds, Dee. 1995. Symbolist Aesthetics and Early Abstract Art: Sites of Imaginary Space. Cambridge: Cambridge University Press. 
POSTPRINT of the article published in 2019 in The Senses and Society, Vol. 14(1), 1-14.

Roholt, Tiger C. 2014. Groove: A Phenomenology of Rhythmic Nuance. New York, NY: Bloomsbury Publishing.

Salimpoor, Valorie N., Mitchel Benovoy, Kevin Larcher, Alain Dagher, and Robert J. Zatorre. 2011. "Anatomically distinct dopamine release during anticipation and experience of peak emotion to music." Nature Neuroscience 14 (2): 257-262.

Schultz, Wolfram. 2016. Dopamine reward prediction error coding. Dialogues in Clinical Neuroscience 18 (1): 23-32.

Solberg, Ragnhild Torvanger, and Alexander Refsum Jensenius. 2017. "Group behaviour and interpersonal synchronization to electronic dance music." Musicae Scientiae, DOI: 1029864917712345.

Stupacher, Jan, Michael J. Hove, Giacomo Novembre, Simone Schütz-Bosbach, and Peter E. Keller. 2013. "Musical groove modulates motor cortex excitability: a TMS investigation." Brain and Cognition 82 (2): 127-136.

Stupacher, Jan, Matthias Witte, and Guilherme Wood. 2017. "Go with the flow: Subjective fluency of performance is associated with sensorimotor synchronization accuracy and stability." In Proceedings of the 25th Anniversary Conference of the European Society for the Cognitive Sciences of Music, Ghent, Belgium.

Sullivan, Philip J., Kate Rickers, and Kimberley L. Gammage. 2014. "The effect of different phases of synchrony on pain threshold." Group Dynamics: Theory, Research, and Practice 18 (2): 122-128.

Swann, Christian, Richard J. Keegan, David Piggott, and Lee Crust. 2012. "A systematic review of the experience, occurrence, and controllability of flow states in elite sport." Psychology of Sport and Exercise 13 (6): 807-819.

Tarr, Bronwyn, Jacques Launay, Emma E.A. Cohen, and Robin I.M. Dunbar. 2015. "Synchrony and exertion during dance independently raise pain threshold and encourage social bonding." Biology Letters 11 (10): 20150767.

Tarr, Bronwyn, Jacques Launay, and Robin I.M. Dunbar. 2014 "Music and social bonding:"self-other" merging and neurohormonal mechanisms." Frontiers in Psychology 5: 1096. 
POSTPRINT of the article published in 2019 in The Senses and Society, Vol. 14(1), 1-14.

Toiviainen, Petri, and Tuomas Eerola. 2003. "Where is the beat? Comparison of Finnish and South African listeners." In Proceedings of the 5th Triennial Conference of the European Society for the Cognitive Sciences of Music, 501-504. Hanover University of Music and Drama, Germany.

Tschacher, Wolfgang, Georg M. Rees, and Fabian Ramseyer. 2014. "Nonverbal synchrony and affect in dyadic interactions." Frontiers in Psychology 5 (1323).

Van Buskirk, Elliot. 2013. People liked their music fastest in the '80s. Spotify Insights. Retrieved on $29^{\text {th }}$ August 2018 from: https://insights.spotify.com/uk/2013/09/19/fastest-pop-music-in-80s/

Vuust, Peter, and Morten L. Kringelbach. 2010. "The pleasure of making sense of music.” Interdisciplinary Science Reviews 35 (2): 166-182.

Vuust, Peter, and Maria A.G. Witek. 2014. "Rhythmic complexity and predictive coding: a novel approach to modeling rhythm and meter perception in music." Frontiers in Psychology, 5 (1111).

Wiltermuth, Scott S., and Chip Heath. 2009. "Synchrony and cooperation." Psychological Science 20 (1): 1-5.

Witek, Maria A.G., Eric F. Clarke, Mikkel Wallentin, Morten L. Kringelbach, and Peter Vuust. 2014. "Syncopation, body-movement and pleasure in groove music." PloS One 9 (4): e94446. 